\title{
CRL4 regulates crown root formation through auxin transport in rice
}

\author{
Yuka Kitomi $^{1}$, Atsushi Ogawa ${ }^{2}$, Hidemi Kitano $^{3}$ and Yoshiaki Inukai ${ }^{1}$ \\ ${ }^{1}$ Graduate School of Bioagricultural Sciences, Nagoya University, Nagoya, Aichi 464-8601, Japan \\ ${ }^{2}$ Department of Biological Production, Akita Prefectural University, Akita, Akita 010-0146, Japan \\ ${ }^{3}$ Bioscience and Biotechnology Center, Nagoya University, Nagoya, Aichi 464-8601, Japan \\ Corresponding author: Y. Inukai, E-mail: inukaiy@agr.nagoya-u.ac.jp, Phone: +81-52-789-4018, \\ Fax: $+81-52-789-4018$
}

Received on March 10, 2008; Accepted on May 26, 2008

\begin{abstract}
Adventitious (crown) roots account for the majority of the root system of monocots. It is reported that auxin plays an important role in the formation of crown roots, but the underlying molecular mechanisms are still unknown. We characterized a rice (Oryza sativa L.) mutant crown rootless4 (crl4) that was found to have defective crown root formation. Besides reduced crown root number, the crl4 mutant showed auxin-related abnormal phenotypical characteristics such as reduced lateral root number and impaired root gravitropism. CRL4 encodes a protein highly homologous with Arabidopsis GNOM, which mediates auxin-dependent plant growth by coordinating the polar localization of auxin efflux carrier PIN1. In the crl4 mutant, auxin transport was impaired in shoots and roots. Besides, the GUS staining controlled by DR5 promoter in the node of crl4 mutant was fainter and spread wider than that of wild-type. These results indicate that maintaining an appropriate auxin accumulation and gradient through CRL4 in the basal portion of shoots is essential for crown root formation in rice.
\end{abstract}

Keywords: auxin transport, crown root, mutant, rice (Oryza sativa L.)

\section{Introduction}

The root system of most dicot plants usually develops from the radicle, while monocot plants have a fibrous root system, characterized by numerous adventitious (crown) roots (Klepper 1992). Crown and lateral roots develop postembryonically from differentiated cells, while a radicle develops during embryogenesis. The phytohormone auxin is essential for root development.
Exogenous treatment with auxin induces ectopic formation of lateral and adventitious roots (Schiefelbein 2003). The transmission of auxin signaling is controlled by the interaction between AUX/IAA and ARF proteins (Liscum and Reed 2002). An auxin signal captured by TIR1, an auxin receptor (Tan et al. 2007), promotes the ubiquitination of IAA protein, a repressor of the auxin responsive transcription, through the SCF ${ }^{\mathrm{TIR} 1}$ complex (Gray et al. 2001). The ubiquitinated IAA proteins are then degraded by the $26 \mathrm{~S}$ proteasome, which allows the auxin responsive transcription to be regulated by ARF proteins; these proteins then act as transcriptional activators or repressors (Gray et al. 2001).

Some mutants in Arabidopsis showed the morphological abnormalities in lateral roots, and they were particularly resulted from stabilized IAA protein. The number of lateral roots was reduced in iaal/axr 5 , iaa3/shy2, iaa12/bdl, iaa14/slr, iaa19/msg2, and iaa28, and increased in iaa7/axr2 and iaa17/axr3 (Leyser et al. 1996, Rouse et al. 1998, Tian and Reed 1999, Nagpal et al. 2000, Rogg et al. 2001, Fukaki et al. 2002, Hamann et al. 2002, Tatematsu et al. 2004, Yang et al. 2004). Recently, it was also reported that auxin-related mutants show abnormalities in root formation in rice, e.g., crll/arll has few crown roots, and that $C R L 1 / A R L 1$ encodes plant-specific AS2/LOB transcriptional factors, which act downstream of IAA and ARF-mediated auxin signaling pathways (Inukai et al. 2005, Liu et al. 2005). Similarly, lateral root initiation was strongly inhibited in the arf7 $\operatorname{arfl} 19$ double mutant (Okushima et al. 2005).

On the other hand, endogenous auxin, indole-3-acetic acid (IAA) is synthesized at the shoot apical meristem and in young leaves, and then transported to the basal portion of shoots and tip of roots. Molecular genetic studies using Arabidopsis

Kitomi Y, Ogawa A, Kitano H, Inukai Y 2008 CRL4 regulates crown root formation through auxin transport in rice. Plant Root 2: 19-28. doi:10.3117/plantroot.2.19

Copyrights 2008, Plant Root (JSRR), www.plantroot.org 
mutants have revealed that a balance between influx and efflux in the auxin transport system is essential for lateral root initiation, and subsequently for primordial development (Fukaki et al. 2007). However, crown root formation has not been studied in detail due to the shortage of monocot mutants.

Here, we report on a newly isolated rice crown rootless 4 ( $\mathrm{crl} 4$ ) mutant that was found to have defective crown root formation. The crl4 mutant showed severe defects not only in crown root but also in lateral root formation. We suspect that CRL4 is involved in auxin-related root formation. This study revealed that CRL4 encodes a protein highly homologous with Arabidopsis GNOM responsible for coordinating the polar localization of auxin efflux carrier PIN1 (Steinmann et al. 1999). We also showed that polar auxin transport was impaired and that auxin accumulation and distribution in the basal portion of shoots was distorted in accordance with the reduction of auxin transport ability in the $\mathrm{crl} 4$ mutant. These results indicate that maintaining an appropriate auxin accumulation and gradient through CRL4 in the basal portion of shoots is essential for crown root formation in rice.

\section{Materials and Methods}

\section{Plant growth conditions}

The crl4 mutant was derived from the somaclonal variation associated with the tissue culture. Seedlings of wild-type rice (Oryza sativa cv. Nipponbare), crl4 mutant and $\mathrm{F}_{2}$ plants derived from the cross between crl4 mutant (japonica variety) and Kasalath (indica variety) were grown in a growth chamber at $29.5^{\circ} \mathrm{C}$ under continuous light. These plants were grown in water or $0.8 \%$ agar medium without nutrients. Transgenic plants were grown in Murashige and Skoog medium (Murashige and Skoog 1962) containing 3\% (w/v) sucrose and $0.3 \%$ Gelrite.

\section{Histological analysis}

Plant tissues were fixed in FAA (formaldehyde:acetic acid : $50 \%$ ethanol at $1: 1: 18$ ) for 24 hours at $4{ }^{\circ} \mathrm{C}$ and then dehydrated in a graded ethanol series. Dehydrated samples were embedded in Paraplast plus (Oxford Labware, St. Louis, MO), sectioned into 8 - $\mu \mathrm{m}$-thick sections by using a rotary microtome, and then stained with toluidine blue.

Map-based cloning, sequence alignment and phylogenetic tree construction

To map the CRL4 gene, linkage analysis was performed using $\mathrm{F}_{2}$ plants derived from the cross between crl4 mutant (japonica variety) and Kasalath (indica variety). BLAST search was performed and the predicted protein sequences were clustered using ClustalW, then Tree View was used to generate the phylogenical output.

\section{Plasmid constructs and plant transformation}

For complementation of the crl4 mutation, the wild-type genomic sequence from -4359 to +1314 (taking the translation initiation site as +1 ) was cut off from bacterial artificial chromosome (BAC) clone OSJNBa0056E06 and cloned into pCAMBIA 1300. pDR5::GUS construct were generated as reported previously (Scarpella et al. 2003). The resulting fusion construct was introduced into Agrobacterium tumefaciens strain EHA105 by electroporation. Agrobacterium-mediated transformation of rice was performed as described previously (Hiei et al. 1994). Transgenic plants were selected on media containing $50 \mathrm{mg} \mathrm{L}^{-1}$ hygromycin.

\section{Expression analysis}

cDNA synthesis for semiquantitative RT-PCR was carried out by using an Omniscript RT kit (Qiagen, Valencia, CA), and RNA was extracted by using TRIzol $^{\circledR}$ reagent (Invitrogen, Carlsbad, CA) according to the manufacturer's instructions. PCR was performed essentially as described by the manufacture. The primer sequences were 5'-CTGTGGAGCTTGATGAATACAC-3' and 5'-CAAGCTTCTCAGGCAACAAATG-3' for CRL4, 5'-AGACCATGCAGGAGGTTATCCG-3' and 5'-ACAACACCAAATCCACCTCCCA-3' for CRL4-like and 5'-GACTACATACAACTCCATCATG-3' and 5'-AGCATTTCCTGTGCACAATGG-3' for $A C T 1$.

\section{In situ hybridization}

In situ hybridization was performed as previously described (Kouchi and Hata 1993). For the CRL4 probe, a 695-nt fragment cut off by Sac I from the complementation construct was subcloned into pBluescript $^{\circledR}$ II KS (-) (Stratagene, La Jolla, CA). Hybridization conditions were at $55^{\circ} \mathrm{C}$ overnight and the probe was mounted $0.2 \mu \mathrm{l}$ per slide.

\section{Auxin transport and accumulation assays}

Auxin transport and accumulation were measured as described by Chhun et al. (2007) with some modifications. To measure the auxin transport ability in shoot, the shoots of 7-day-old seedlings were decapitated and the cut stem of $1.2 \mathrm{~cm}$ in length was applied the vaseline paste containing $1 \mu \mathrm{M} 3-\left[5(n)-{ }^{3} \mathrm{H}\right]-$ 
Indolylacetic acid $\left(\left[^{3} \mathrm{H}\right]-\mathrm{IAA}\right.$, Amarsham, USA). After 4 hours incubation at $28^{\circ} \mathrm{C}$ by water culture, the $3 \mathrm{~mm}$ segments were cut from $3 \mathrm{~mm}$ upper of the basal node. The $1.2 \mathrm{~cm}$ seminal root tips were obtained from 7-day-old seedlings then each was placed to keep the contact of the apical or the basal end of root tip with the vaseline paste containing $1 \mu \mathrm{M}\left[{ }^{3} \mathrm{H}\right]-\mathrm{IAA}$ on a slide glass, for measuring the transport ability in root. After 4 hours incubation at $28^{\circ} \mathrm{C}$ in humid condition, the $3 \mathrm{~mm}$ segments were cut from the basal or apical

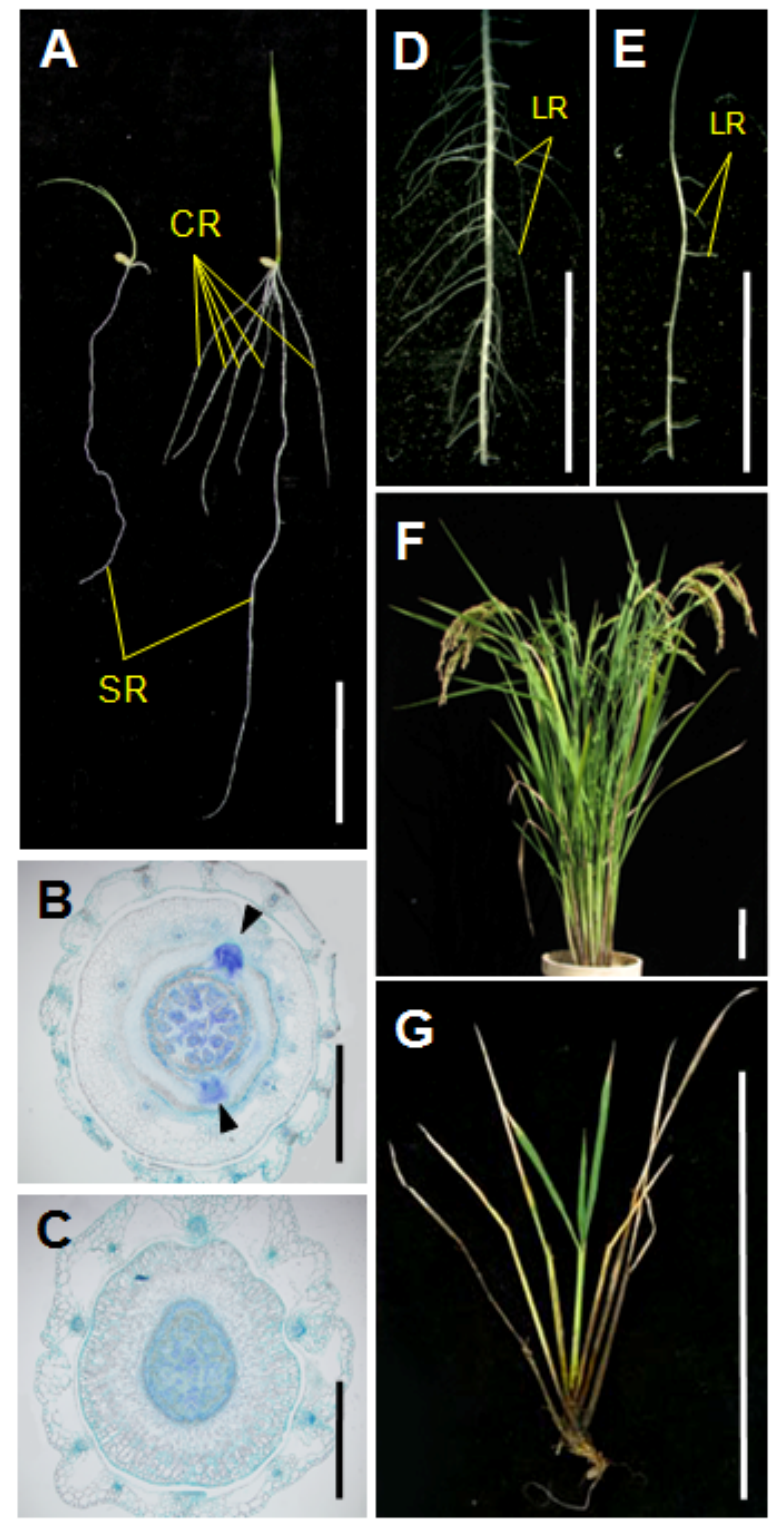

Fig. 1. Phenotypes of crl4. (A) 2-week-old wild-type, Nipponbare (right) and crl4 (left) seedlings. CR, crown root; SR, seminal root. Bar $=10 \mathrm{~cm}$. (B) and (C) Cross sections through the nodes in 5-day-old wild-type (B) and crl4 (C) seedlings. The arrowheads indicate the crown root primordia. Bars $=500 \mu \mathrm{m}$. (D) and (E) Lateral roots in 2-week-old wild-type (D) and crl4 (E) seedlings. LR, lateral roots. Bars $=1 \mathrm{~cm}$. $(\mathrm{F})$ and $(\mathrm{G})$ 4-month-old wild-type $(\mathrm{F})$ and $\mathrm{crl} / \mathrm{G})$. Bars $=10 \mathrm{~cm}$. end of the root tip. Each sample was placed into $5 \mathrm{ml}$ of scintillation fluid and radioactivity was counted with the scintillation counter (LSC-5100, Alaka). To measure the auxin accumulation, the shoots of 5-day-old seedlings were decapitated and the cut stem of $1 \mathrm{~cm}$ in length was applied the vaseline paste containing $1 \mu \mathrm{M}\left[{ }^{3} \mathrm{H}\right]-$ IAA. After 4 hours incubation at $28^{\circ} \mathrm{C}$, the $3 \mathrm{~mm}$ segments of the basal end of shoot and whole seminal root were cut and placed into $5 \mathrm{ml}$ of scintillation fluid. Radioactivity was counted with the scintillation counter.

\section{Results}

\section{Characterization of the crl4 mutant}

Two-week-old wild-type plants (Nipponbare) formed several crown roots in comparison to $\mathrm{crl} / 4$ mutants, in which crown root production was scarce at the same developmental stage (Fig. 1A). We cross-sectioned the nodes in 5-day-old wild-type and crl4 mutant seedlings to determine the cause of crown root reduction in crl4 mutants. In wild-type, several crown root primordia were formed on the outside, adjacent to the peripheral vascular cylinder (Fig. 1B). In contrast, crl4 mutants did not produce any crown root primordia (Fig. 1C). Besides the reduced crown root number, the number of lateral roots that were obtained from a seminal root of the crl4 mutant was clearly lower than that of the wild-type (Fig. 1D and 1E). The seminal root in the crl4 mutant was shorter than that of the wild-type (Fig. 1A), although there were no significant differences in the timing of the emergence of the seminal root. The crl4 mutant produced the higher number of tillers (data not shown) and their growth angles were wider than those of wild-type (Fig. 1F and $1 \mathrm{G})$. Some crl4 mutants survived for more than 4 months. Four-month-old wild-type plants could reach the grain filling stage, but crl4 mutants with tiny shoots and a single seminal root could not make the transition from the vegetative to the reproductive phase (Fig. 1F and 1G).

It has been reported that the reduction of crown and lateral root number are commonly seen in auxin-related phenotypes in rice (Inukai et al. 2005, Liu et al. 2005). The abnormality in gravitropic response has also been reported in several auxin-related mutants in Arabidopsis (Leyser et al. 1996, Tian and Reed 1999, Nagpal et al. 2000, Fukaki et al. 2002, Yang et al. 2004). We examined the root gravitropic response in $\mathrm{crl} 4$ mutants by measuring the curvature after gravistimulation at $90^{\circ}$ to the vertical. Wild-type roots responded sharply to the change in the gravity vector, whereas the response of $\mathrm{crl} 4$ roots was impaired (Fig. 2A). The root tip angle of wild-type and crl4 roots was compared ( $\theta$ in Fig. 2B); all wild-type 


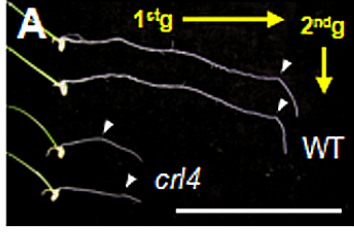

B

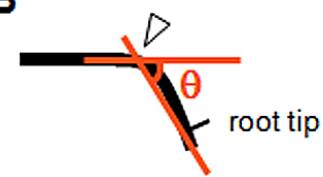

C

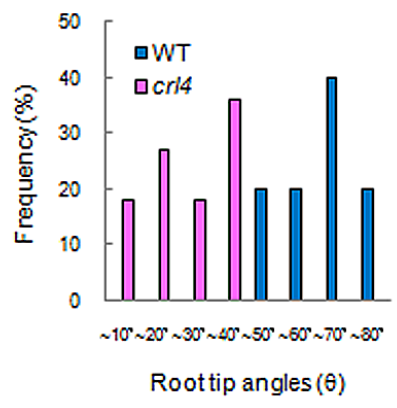

Fig. 2. Gravitropic response in a seminal root of $\mathrm{crl}$. (A) Wild-type and crl4 seedlings were grown vertically (arrow of $1^{\text {st }} \mathrm{g}$ ) and then rotated $90^{\circ}$ (arrow of $2^{\text {nd }} \mathrm{g}$ ). Bar $=$ $10 \mathrm{~cm}$. (B) and (C) The root tip angles $(\theta)$ in (B) was measured 24 hours after reorientation $(\mathrm{C})$.

roots had root tip angles of over $50^{\circ}$, while all $\mathrm{crl} 4$ mutants had angles of below $50^{\circ}$ (Fig. 3C). The gravitropic response was therefore defective in $\mathrm{crl} 4$ mutants. These results suggest that CRL4 has an auxin-related function.

\section{CRL4 encoded a gene similar to GNOM}

We employed a map-based cloning approach to isolate the $C R L 4$ gene. The $C R L 4$ locus was mapped on the long arm of chromosome 3 around $101.9 \mathrm{cM}$. By comparing the nucleotide sequences of the $\mathrm{crl} / 4$ mutant and wild-type, we found a single nucleotide substitution in Os03g46330 on the BAC clone OSJNBa0056E06, which resulted in a nonsense mutation (Fig. 3A). Introduction of a 9.2-kbp genomic DNA fragment containing the entire candidate gene (Fig. 3B) to the crl4 mutant complemented the abnormal crl4 mutant phenotype (Fig. 3C), produced crown roots and lateral roots same as the redifferentiated plant of wild-type (data not shown). Shoot of the complemented $\mathrm{crl} 4$ plant was normal without abnormalities such as the higher number and wider growth angles of tillers than those of wild-type. Therefore, we concluded that $\mathrm{crl} 4$ phenotypes are caused by the loss-of-function mutation in the predicted $C R L 4$ gene.

The CRL4 gene encodes a protein of 1176 amino acid residues in one exon with the Sec7 domain characteristic of GNOM (Fig. 3A). It has been reported that GNOM is a membrane-associated guanine-nucleotide exchange factor on the ADP-ribosylation factor $G$ protein (ARF-GEF) and that ARF-GEFs regulate specific endosomal trafficking pathways (Steinmann et al. 1999, Geldner et al. 2003a). GNOM is required for the recycling of PIN1, which mediates polar auxin transport in Arabidopsis between the endosome and plasma membrane by vesicle trafficking (Geldner et al. 2003a). Thus, it is

\section{A}

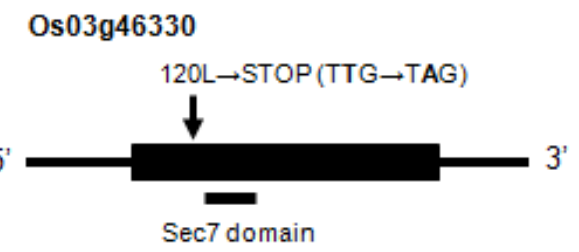

B
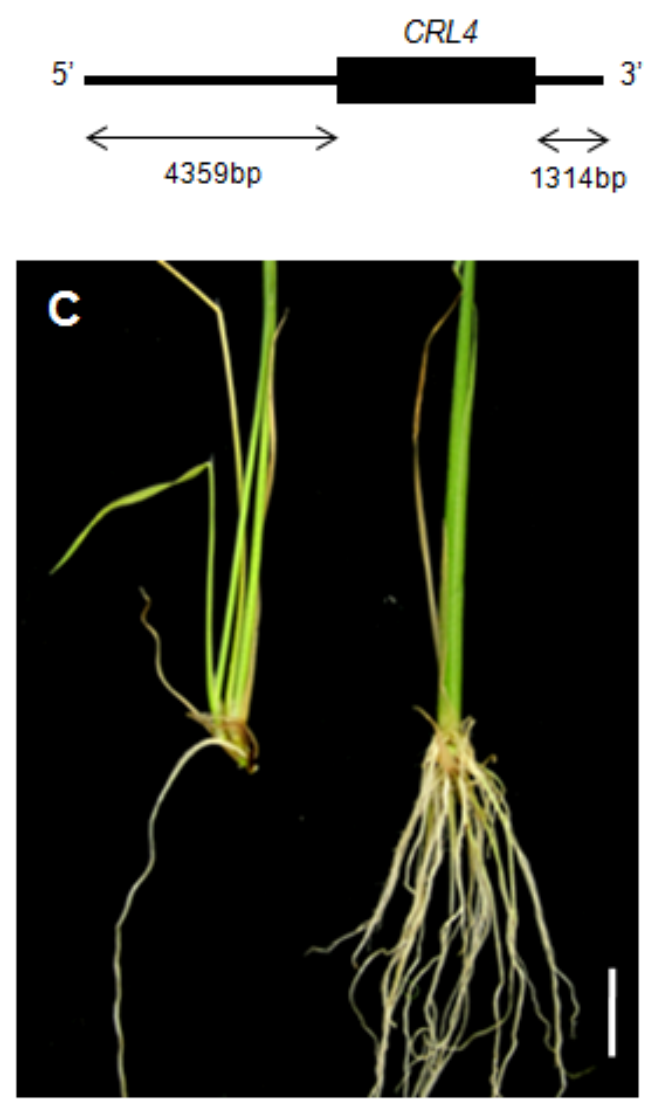

Fig. 3. Map-based cloning and phenotypic complementation by the introduction of CRL4. (A) Structure of CRL4 gene. Black box indicated the exon, the arrow represented a single nucleotide mutation, resulted in the change into stop codon. (B) Structure of the genomic DNA fragment encompassing the entire CRL4 gene. (C) The crl4 mutant plant containing the empty vector (left) and the genomic DNA fragment encompassing the entire CRL4 gene (right) were shown. Bar $=1 \mathrm{~cm}$.

believed that GNOM plays an important role in polar auxin transport through the establishment of coordinated polar localization of PIN1, which in turn highly depends on the directed vesicle trafficking (Steinmann et al. 1999, Geldner et al. 2003a). We performed a BLAST search using the amino acid sequence of CRL4 and found its homologous gene CRL4-like 
(Os02g22090) on chromosome 2 in rice (Fig. 4A). CRL4-like shared $83 \%$ amino identity in Sec7 domain with CRL4, while GNOM shared 90\% (Fig. 4A). Then we drew dendogram of CRL4, CRL4-like and Arabidopsis GNOM family members; GNOM, GNOM-like1 (GNL1) and GNOM-like2 (GNL2). It was constructed based on the amino acid sequence of the Sec7 domains and GBF1, which is human ARF-GEF, was used as outgroup. It therefore appears that CRL4 is more homologous with GNOM in Arabidopsis than CRL4-like in rice (Fig. 4B).
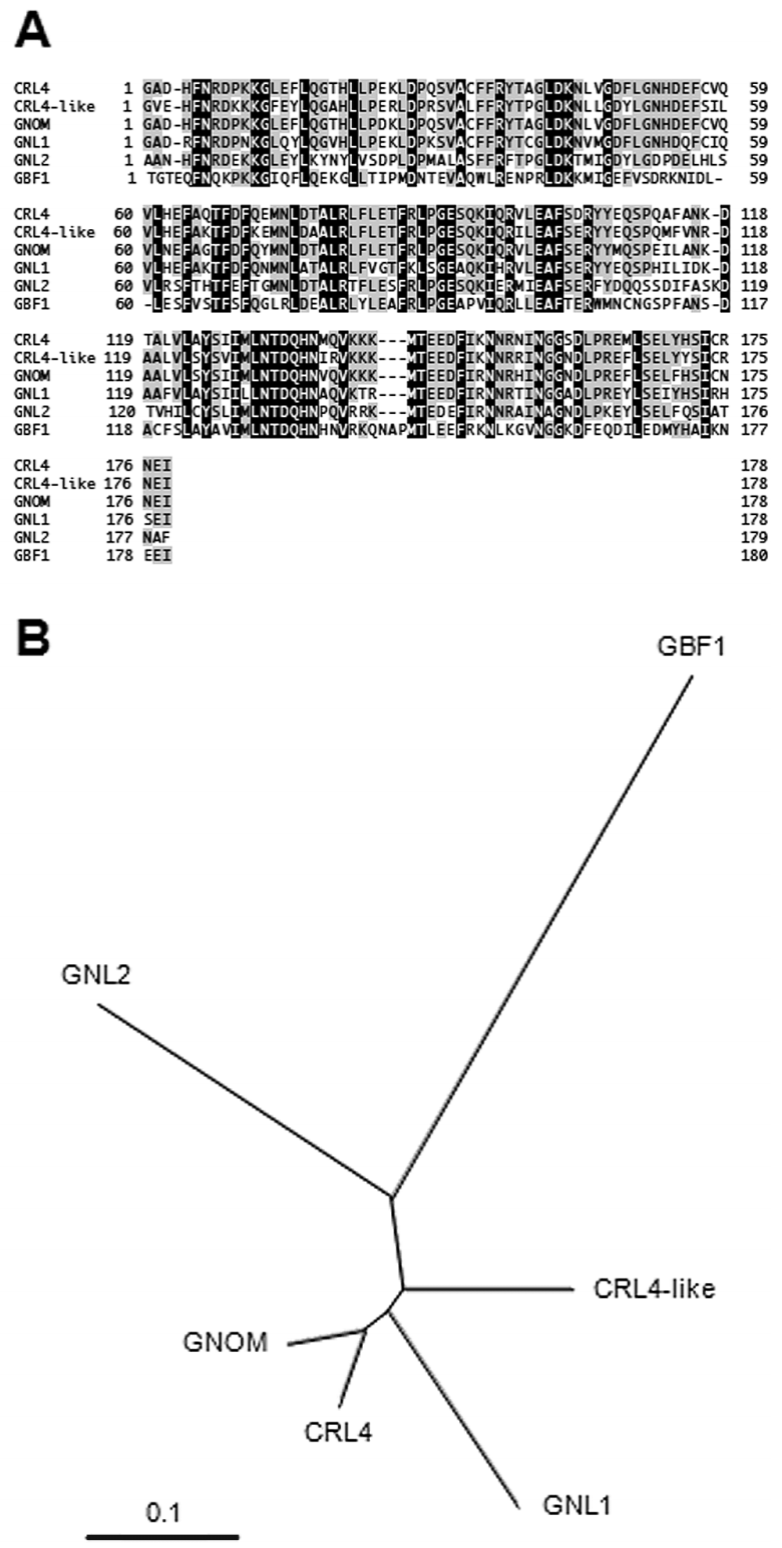

Fig. 4. Structure of CRL4. (A) Comparison of amino acid sequence of Sec7 domain in CRL4 with that in CRL4-like, GNOM, GNL1 and GNL2. (B) Dendogram of the GNOM family proteins of Sec7 domain in Arabidopsis (GNOM, GNL1 and GNL2) and rice (CRL4 and CRL4-like).
Analysis of CRL4 expression and auxin distribution

We examined the expression of $C R L 4$ in various organs. To estimate CRL4 transcript levels, we performed semi-quantitative RT-PCR analysis (Fig. 5A). $C R L 4$ was expressed in all the examined organs; high levels of $C R L 4$ transcripts were found to be accumulated in leaf blades and roots. CRL4-like was also expressed in all the organs and the expression pattern was similar to that of $C R L 4$ (Fig. 5A).

We also examined the $C R L 4$ expression pattern by in situ hybridization. In the stem, signals were observed at the steles of the crown root primordia (Fig. 5B and 5C), the vascular bundles and the parenchyma cells adjacent to the peripheral vascular cylinder of the stem where the crown root primordia were formed (Fig. 5D and 5E). In addition, we compared the GUS staining controlled by DR5 promoter between wild-type and crl4 mutant. DR5 is often used as an artificial promoter responding to auxin signals in Arabidopsis (Sabatini et al. 1999). This promoter was also used as a marker to visualize the in vivo distribution of auxin in rice (Scarpella et al., 2003). The staining was observed at the tip and middle of leaf blade, the leaf sheath and the basal portion of the shoot in wild-type plant (Fig. 5F). In the crl4 mutant, faint staining was observed at the tip of leaf blade and the basal portion of the shoot (Fig. 5F and 5G). Then we made the cross section of the stem of pDR $5:: G U S$ transgenic plant. The GUS staining was localized at the vascular bundles and the parenchyma cells adjacent to the peripheral vascular cylinder in wild-type (Fig. 5H). On the other hand, the GUS staining was scarcely observed at the vascular bundles and faint staining spread the whole parenchyma cells in $\mathrm{crl} 4$ mutant (Fig. 5I). The regions where the localized GUS staining of $p D R 5:: G U S$ in wild-type was observed coincided with the regions where $C R L 4$ was expressed (Fig. 5D and 5H). It has previously been reported that OsPIN1 is also expressed in these regions (Xu et al. 2005). GNOM coordinates the localization of PIN1, which is responsible for establishing the auxin gradient in Arabidopsis (Steinmann et al. 1999), and CRL4 was expressed in the vascular bundles and the predicted region where the crown root primordia were formed in rice. These observations indicate that the similarity between CRL4 function in rice and GNOM function in Arabidopsis.

\section{Defective auxin transport in crl4 mutants}

It is well known that polar auxin transport is involved in vascular formation (Berlenth and Mattsson 2000) and that impaired auxin flow adversely affects vascular development (Koizumi et al. 2000, Geldner et al. 2003b, Sieburth et al. 2006). Abnormal vascular 
structure was observed in the seminal root of the $\mathrm{crl} 4$ mutant. Metaxylem elements were scattered in a regular pattern around the steles in wild-type, whereas they were found to be increased and arranged in a
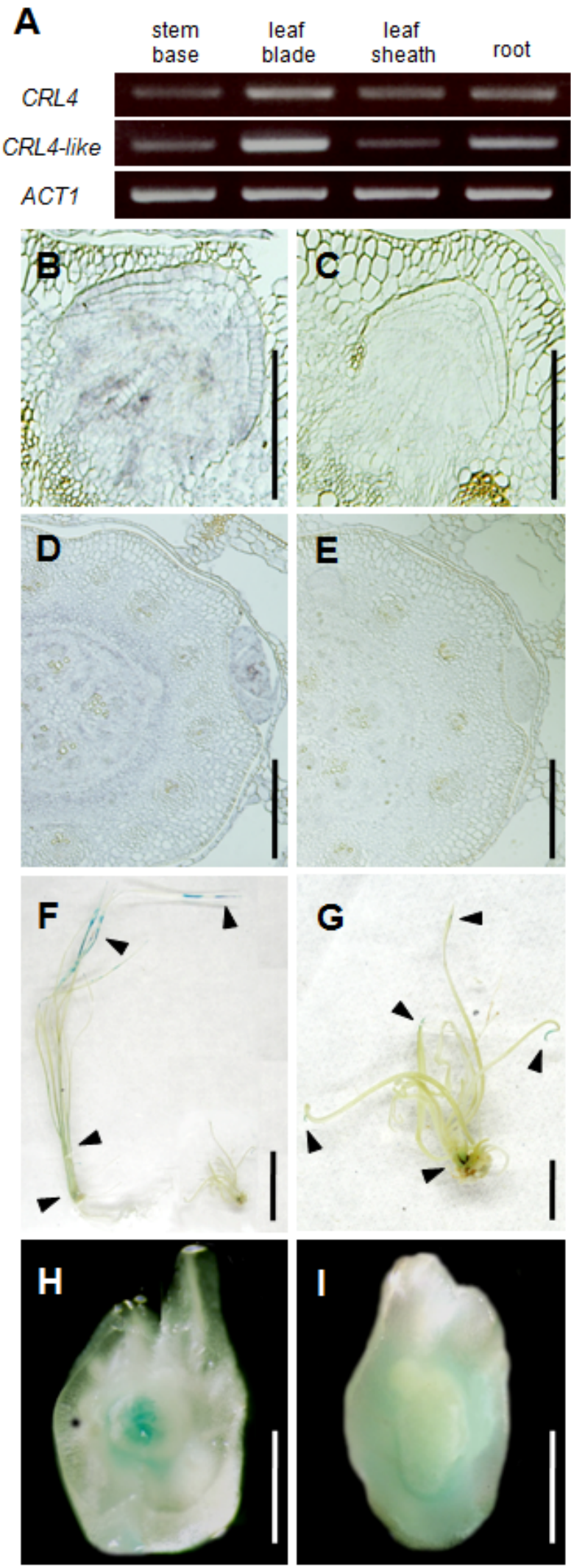

radial array around the steles in crl4 mutants (Fig. 6A and $6 \mathrm{~B}$ ).

As mentioned above, CRL4 encodes a protein highly homologous with Arabidopsis GNOM, which mediates auxin-dependent plant growth by coordinating the polar localization of PIN1. In addition, it is reported that wild-type rice plants rarely produce crown roots with 1- $N$-naphthylphthalamic acid (NPA) treatment, an inhibitor of polar auxin transport (Liu et al. 2005, Morita and Kyozuka 2007). Phenotypes of wild-type rice seedlings treated with NPA mimicked those of $\mathrm{crl} 4 \mathrm{mutants}$. These reports indicate that the polar auxin transport is indispensable for crown root formation in rice and that the abnormalities in the $\mathrm{crl} 4$ mutant are a result of impaired polar auxin transport.

We compared the auxin transport kinetics in $\mathrm{crl} 4$ mutants with those in wild-type using radioactive auxin $\left[{ }^{3} \mathrm{H}\right]$-IAA and found that the transport efficiency of $\left[{ }^{3} \mathrm{H}\right]$-IAA in $\mathrm{crl} 4$ mutant shoots was reduced to $17 \%$ of that of wild-type shoots (Fig. 6C). Next, we assessed the acropetal (to root tip) and basipetal (from root tip) transport systems in a seminal root and found that in crl4 mutants, acropetal and basipetal transport activities were $41 \%$ and $48 \%$, respectively, compared with those observed in wild-type (Fig. 6C). We also investigated the accumulation of $\left[{ }^{3} \mathrm{H}\right]-\mathrm{IAA}$ at seminal root and node where crown roots are produced and found that the accumulation in crl4 seminal root was reduced to $13 \%$ of that in wild-type (Fig. 6D). It was also revealed that $\left[{ }^{3} \mathrm{H}\right]-\mathrm{IAA}$ itself was scarcely transported to the root in $\mathrm{crl} / 4$ mutants. Meanwhile, the accumulation in the nodes of $\mathrm{crl} 4$ mutants was also decreased to $53 \%$ of that of wild-type (Fig. 6D). These results suggest that auxin transport is defective in $\mathrm{crl} 4$ mutants and distorted auxin transport results in the reduced auxin accumulation in the node and the seminal root.

Fig. 5. Expression patterns of $C R L 4$ and auxin distribution. (A) CRL4 and CRL4-like expression in various organs of wild-type rice. Semiquantitative RT-PCR was concluded, and $A C T 1$ was used as a control. Stem base includes shoot apical meristem and nodes. (B) to (E) In situ hybridization with cross sections through the nodes in 7-day-old wild-type seedling. Localization of CRL4 in the crown root primordia with the antisense probe (B) and with the sense probe $(\mathrm{C})$. Bars $=100 \mu \mathrm{m}$. Localization of $C R L 4$ in the node developing crown root primordia with antisense probe (D) and with sense probe (E). Bars $=200 \mu \mathrm{m}$. No signal was detected with sense probe of CRL4. (F) to (I) Localized GUS staining controlled by DR5 promoter. Whole plant of wild-type (left) and crl4 mutant (right) (F). Bar $=5 \mathrm{~cm}$. Magnified the whole $\mathrm{crl} 4$ mutant in Fig. $5 \mathrm{E}(\mathrm{G})$. Bar $=1 \mathrm{~cm}$. The black arrowheads indicate the localized GUS stainings. Cross sections through the nodes in wild-type $(\mathrm{H})$ and $\mathrm{crl} 4$ (I) transgenic plant. Bars $=1 \mathrm{~mm}$. 


\section{Discussion}

\section{Effect of crl4 mutation in rice}

CRL4 protein is highly homologous with the Arabidopsis GNOM protein, which has been shown contribute to the apical-basal pattern formation in Arabidopsis embryogenesis (Mayer et al. 1993). Strong alleles of gnom show loss of cell-to-cell alignment along the embryonic axis, lack an embryonic root, and display variable levels of fusion or deletion of cotyledons and hypocotyls (Mayer et al. 1993). Weak alleles of gnom have fused cotyledon with lost lateral veins and clustered tracheary elements, and rosette leaves whose venations become more complicated with the increase in number of freely ending veinlets (Koizumi et al. 2000, Geldner et al. 2003b, Sieburth et al. 2006). These reports indicate that gnom mutation causes serious defects in plant development. Nonsense mutation in CRL4 occurred before the Sec7 domain characteristic of GNOM, suggesting that CRL4 lost its function in $\mathrm{crl} 4$ mutants.

The crl4 mutant showed several abnormalities such as loss of crown roots, aberrant gravitropic response, and increase of metaxylem-like structures in the seminal root. In addition, it could not make the transition from the vegetative to the reproductive phase. However, these abnormalities in crl4 mutants did not seem as severe as those observed in severe alleles of gnom (Mayer et al. 1993, Shevell et al. 1994). The phenotypes of crl4 mutant was similar to weak alleles of gnom (Geldner et al. 2003b), nevertheless the crl4 mutant shows a loss in most of the CRL4 functions. GNOM has two homologous genes, GNL1 and GNL2, in Arabidopsis, but it is reported that GNL1 does not contribute much to the polar auxin transport (Richter et al. 2007, Teh and Moore 2007). Therefore, the absence of genes with redundant function may result in severe mutant phenotypes of gnom. In rice, we hypothesized that a homologous gene of CRL4, CRL4-like, plays a redundant role with $C R L 4$ and can partially compensate for $C R L 4$ function because the expression pattern of CRL4-like is identical to that of CRL4 (Fig. 5A). Besides, CRL4-like was also considered to play a redundant role in embryogenesis because of the absence of abnormalities in the mature embryo of the crl4 mutant (data not shown).

\section{Defective polar auxin transport in crl4 mutants}

It is believed that GNOM plays an important role in polar auxin transport through the establishment of coordinated polar localization of PIN1 in Arabidopsis (Steinmann et al. 1999, Geldner et al. 2003a). Since polar auxin transport is essential for plant organization,
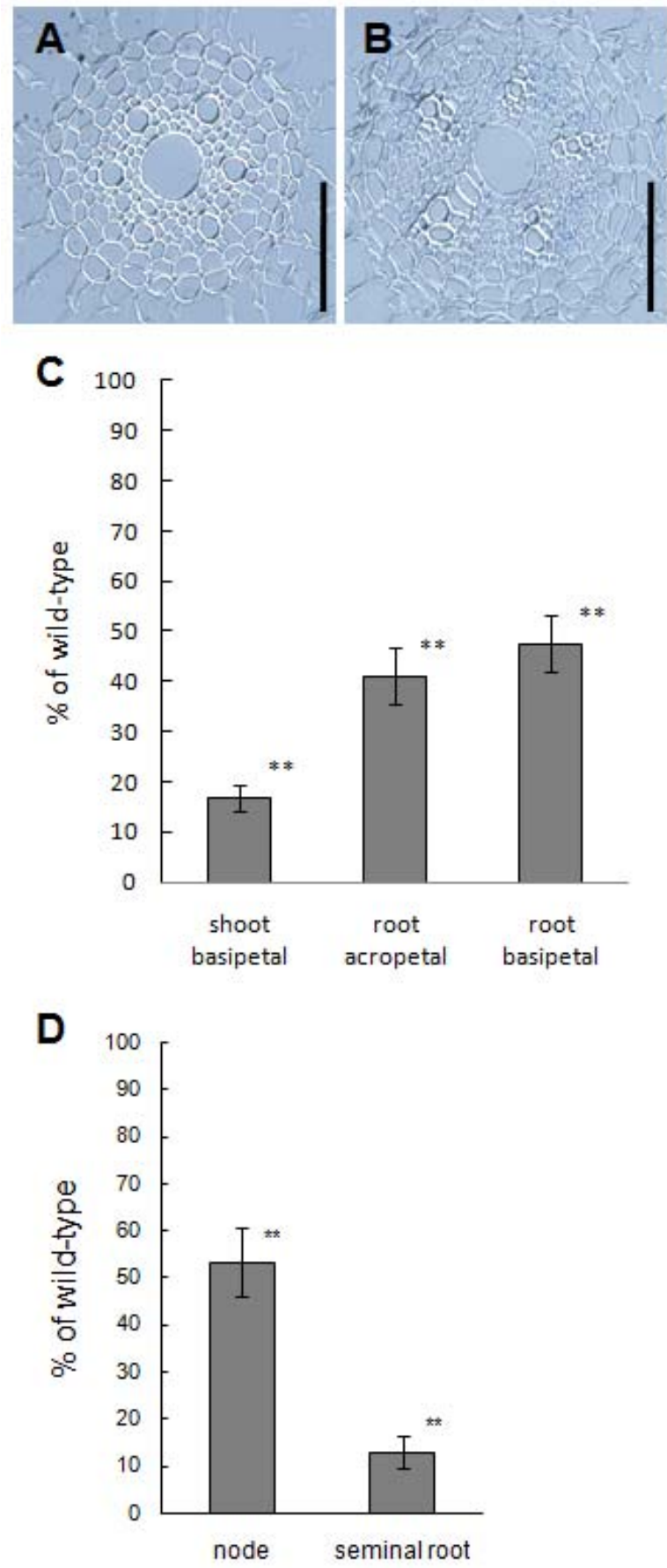

Fig. 6. Comparison of auxin transport of $\mathrm{crl} 4$ with that of wild-type. (A) and (B) Cross section of the basal part of the 7-day-old wild-type seminal root (A) and $\operatorname{crl} 4$ (B). Bars $=50$ $\mu \mathrm{m}$. (C) Comparison of the ability of $\left[{ }^{3} \mathrm{H}\right]-\mathrm{IAA}$ transport. Shoot besipetal (from apex to base) transport, root acropetal (from base to root tip) transport and root basipetal (from root tip to base) transport were measured. The measured value of $\left[{ }^{3} \mathrm{H}\right]$-IAA was expressed as a percentage relative to wild-type. $* *$ indicates significantly different from wild-type at $1 \%$. (D) Comparison of the accumulation of transported $\left[{ }^{3} \mathrm{H}\right]$-IAA from shoot to node and seminal root. The measured value of $\left[{ }^{3} \mathrm{H}\right]$-IAA was expressed as a percentage relative to wild-type. $* *$ indicated significantly different from wild-type at $1 \%$. 
gnom mutants show several defects in cell polarity and embryo axis formation (Steinmann et al. 1999). Formation of lateral roots is regulated by auxin transported acropetally from above-ground tissues to the root tip and basipetally from the root tip (Geldner et al. 2003b, Smet et al. 2007). Lateral root formation was found to be inhibited in the weak alleles of gnom (Geldner et al. 2003b).

Both basipetal transport in the shoot and acropetal transport in the seminal root of crl4 mutants were lower than those observed in wild-type. Because it has been reported that these polar transport mechanisms are mainly mediated by auxin efflux carriers (Jacobs and Gilbert 1983), these findings appear reasonable with regard to the predicted CRL4 function, which suggests that CRL4 might be related to auxin efflux carriers such as GNOM in Arabidopsis. Basipetal transport in the seminal root tip of crl4 mutants was also lower than that of wild-type, suggesting that CRL4 is also required for auxin basipetal transport in root tips. The lateral root number was decreased in crl4 mutants (Fig. 1D and 1E) and this fact is consistent with the finding that weak alleles of gnom fail to produce lateral roots due to insufficient basipetal transport in the seminal root tip (Geldner et al. 2003b). These results indicate that CRL4 is necessary for the normal development of roots and shoots through mediation of a viable auxin transport system.

Compared with wild-type, $\left[{ }^{3} \mathrm{H}\right]-\mathrm{IAA}$ accumulation in the basal portion of shoots was reduced to $53 \%$ in the crl4 mutant (Fig. 6D). From the GUS staining controlled by DR5 promoter, the staining was observed at the tip and middle of leaf blade, the leaf sheath and the basal portion of the shoot in wild-type plant (Fig. 5F). On the other hand, faint staining was only observed at the tip of leaf blade and the basal portion of the shoot in the $\mathrm{crl} / 4$ mutant (Fig. 5F and $5 \mathrm{G})$. Since the tip of leaf blade is one of the auxin biosynthesis sites, it was conceivable that the absent of the GUS staining in the middle of leaf blade and the leaf sheath in crl4 mutant resulted from the auxin transport inhibition in it. At the stem, auxin was localized at the vascular bundles and the parenchyma cells adjacent to the peripheral vascular cylinder in wild-type (Fig. 5H) but not in crl4 mutant; the GUS staining was scarcely observed at the vascular bundles and faint staining spread the whole parenchyma cells in crl4 mutant (Fig. 5I). When the auxin gradient is lost due to auxin transport inhibition and auxin is distributed equally, lateral root initiation ceases to occur in Arabidopsis roots (Smet et al. 2007). That is, it indicated that CRL4 plays an important role in crown root initiation through the establishment of appropriate auxin accumulation and gradient. In addition, formation of the lateral root primordia necessitates the re-establishment of cell division and elongation patterns orthogonal to the axis of the primary root in Arabidopsis (Geldner et al. 2003b). It is necessary for PIN1 to shift its polarity orthogonally in the daughter cells of stage I primordia in order to make the axis of the lateral root primordia orthogonal to that of the primary root. It is suggested that GNOM is also critical for the switching of PIN1 re-localization in the daughter cells of stage I primordia (Geldner et al. 2003b). The regions expressing CRL4 coincided with the regions stained by pDR5::GUS and $p O s P I N 1: \because G U S$ in rice (Fig. $5 \mathrm{D}$ and $5 \mathrm{H}$, Xu et al. 2005), indicating that CRL4 also plays a role in crown root development after the initiation. Besides, lateral root development was impaired in $\mathrm{crl} 4$ mutants (Fig. 1D and 1E). Therefore, CRL4 is considered to regulate both root initiation and development by similar way to GNOM function. In conclusion, these findings indicate that an appropriate auxin accumulation and gradient mediated through CRL4 function is essential for the formation of crown and lateral roots in rice.

\section{Acknowledgements}

We thank Dr. Takashi Sazuka, Dr. Tory Chhun and Dr. Kotaro Miura of bioscience and biotechnology center of Nagoya University for their valuable technical supports. This work was funded by the Grant-in-Aid from the Japan Society for the Promotion of Science (No. 18780002).

\section{References}

Berleth T, Mattsson J 2000 Vascular development: tracing signals along veins. Curr. Opin. Plant Biol. 3: 406-411.

Chhun T, Uno Y, Taketa S, Azuma T, Ichii M, Okamoto T, Tsurumi S 2007 Saturated humidity accelerates lateral root development in rice (Oryza sativa L.) seedling by increasing phloem-based auxin transport. J. Exp. Bot. 58: 1695-1704.

Fukaki H, Tameda S, Masuda H, Tasaka M 2002 Lateral root formation is blocked by a gain-of-function mutation in the SOLITARY-ROOT/IAA14 gene of Arabidopsis. Plant J. 29: 153-168.

Fukaki H, Okushima Y, Tasaka M 2007 Auxin-mediated lateral root formation in higher plants. Int. Rev. Cytol. 256: 111-137.

Geldner N, Anders N, Wolters H, Keicher J, Kornberger W, Muller P, Delbarre A, Ueda T, Nakano A, Jürgens G 2003a The Arabidopsis GNOM ARF-GEF mediates endosomal recycling, auxin transport, and auxin-dependent plant growth. Cell 112: 219-230.

Geldner N, Richter S, Vieten A, Marquardt S, Torres-Ruiz RA, Mayer U, Jürgens G 2003b Partial loss-of-function alleles reveal a role for GNOM in auxin transport-related, post-embryonic development of Arabidopsis. Development 131: 389-400.

Gray WM, Kepinski S, Rouse D, Leyser O, Estelle M 2001 Auxin regulates the $\mathrm{SCF}^{\mathrm{TIR} 1}$-dependent degradation of AUX/IAA proteins. Nature 414: 271-276. 
Hamann T, Benková E, Baurle I, Kientz M, Jürgens G 2002 The Arabidopsis BODENLOS gene encodes an auxin response protein inhibiting MONOPTEROS-mediated embryo patterning. Genes Dev. 16: 1610-1615.

Hiei Y, Ohta S, Komari T, Kumashiro T 1994 Efficient transformation of rice (Oryza sativa L.) mediated by Agrobacterium and sequence analysis of the boundaries of the T-DNA. Plant J. 6: 271-282.

Inukai Y, Sakamoto T, Ueguchi-Tanaka M, Shibata Y, Gomi K, Umemura I, Hasegawa Y, Ashikari M, Kitano H, Matsuoka M 2005 Crown rootless 1, which is essential for crown root formation in rice, is a target of an AUXIN RESPONSE FACTOR in auxin signaling. Plant Cell 17: 1387-1396.

Jacobs M, Gilbert SF 1983 Basal localization of the presumptive auxin carrier in pea stem cells. Science 220: 1297-1300.

Klepper B 1992 Development and growth of crop root systems. In: Hatfield JL, Stewart BA, Eds., Limitations to plant root growth. Springer-Verlag, Berlin, pp. 265-286.

Koizumi K, Sugiyama M, Fukuda H 2000 A series of novel mutants of Arabidopsis thaliana that are defective in the formation of continuous vascular network: calling the auxin signal flow canalization hypothesis into question. Development 127: 3197-3204.

Kouchi H, Hata S 1993 Isolation and characterization of novel nodulin cDNAs representing genes expressed at early stages of soybean nodule development. Mol. Gen. Genet. 238: 106-119.

Leyser HM, Pickett FB, Dharmasiri S, Estelle M 1996 Mutations in the AXR3 gene of Arabidopsis result in altered auxin response including ectopic expression from the SAUR-AC1 promoter. Plant J. 10: 403-413.

Liscum E, Reed JW 2002 Genetics of AUX/IAA and ARF action in plant growth and development. Plant Mol. Biol. 49: 387-400.

Liu H, Wang S, Yu X, Yu J, He X, Zhang S, Shou H, Wu P 2005 ARL1, a LOB domain protein required for adventitious root formation in rice. Plant J. 43: 47-56.

Mayer U, Buttner G, Jürgens G 1993 Apical-basal pattern formation in the Arabidopsis embryo: studies in the role of the gnom gene. Development 117: 149-162.

Morita Y, Kyozuka J 2007 Characterization of OSPID, the rice ortholog of PINOID, and its possible involvement in the control of polar auxin transport. Plant Cell Physiol. 48: 540-549.

Murashige T, Skoog F 1962 A revised method for rapid growth and bioassays with tobacco tissue cultures. Physiol. Plant. 15:472-497

Nagpal P, Walker LM, Young JC, Sonawala A, Timpte C, Estelle M, Reed JW 2000 AXR2 encodes a member of the Aux/IAA protein family. Plant Physiol. 123: 563-573.

Okushima Y, Overvoorde PJ, Arima K, Alonso JM, Chan A, Chang C, Ecker JR, Hughes B, Lui A, Nguyen D, Onodera C, Quach H, Smith A, Yu G, Theologis A 2005 Functional genomic analysis of the AUXIN RESPONSE FACTOR gene family members in Arabidopsis thaliana: unique and overlapping functions of ARF7 and ARF19. Plant Cell 17: 444-463.

Richter S, Geldner N, Schrader J, Wolters H, Stierhof YD, Rios G, Koncz C, Robinson DG, Jürgens G 2007 Functional diversification of closely related ARF-GEFs in protein secretion and recycling. Nature 448: 488-492

Rogg LE, Lasswell J, Martel B 2001 A gain-of-function mutation in IAA28 suppresses lateral root development. Plant Cell 13: 465-480.

Rouse D, Mackay P, Stirnberg P, Estelle M, Leyser O 1998
Changes in auxin response from mutations in an AUX/IAA gene. Science 279: 1371-1373.

Sabatini S, Beis D, Wolkenfelt H, Murfett J, Guilfoyle T, Malamy J, Benfey P, Leyser O, Bechtold N, Weisbeek P, Scheres B 1999 An auxin-dependent distal organizer of pattern and polarity in the root. Cell 99: 463-472.

Scarpella E, Rueb S, Meijer AH 2003 The RADICLELESS1 gene is required for vascular pattern formation in rice. Development 130: 645-658.

Schiefelbein J 2003 Cell-fate specification in the epidermis: a common patterning mechanism in the root and shoot. Curr Opin. Plant Biol. 6: 74-78.

Shevell DE, Leu W, Gilimor CS, Xia G, Feldmann KA, Chua N 1994 EMB30 is essential for normal cell division, cell expansion, and cell adhesion in Arabidopsis and encodes a protein that has similarity to Sec7. Cell 77: 1051-1062.

Sieburth LE, Muday GK, King EJ, Benton G, Kim S, Metcalf KE, Meyers L, Seamen E, Norman JMV 2006 SCARFACE encodes ARF-GAP that is required for normal auxin efflux and vein patterning in Arabidopsis. Plant Cell 18: 1396-1411.

Smet ID, Tetsumura T, Rybel BD, Frey NF, Laplaz, L, Casimiro I, Swarup R, Naudts M, Vanneste S, Audenaert D, Inze D, Bennett MJ, Beeckman T 2007 Auxin-dependent regulation of lateral root positioning in the basal meristem of Arabidopsis. Development 134: 681-690.

Steinmann T, Geldner N, Grebe M, Mangold S, Jackson CL, Paris S, Galweiler L, Palme K, Jürgens G 1999 Coordinated polar localization of auxin efflux carrier PIN1 by GNOM ARF GEF. Science 286: 316-318.

Tan X, Calderon-Villalobos LIA, Sharon M, Zheng C, Robinson CV, Estelle M, Zheng N 2007 Mechanism of auxin perception by the TIR1 ubiquitin ligase. Nature 446: 640-645.

Tatematsu K, Kumagai S, Muto H, Sato A, Watahiki M, Harper RM, Liscum E, Yamamoto K 2004 Massugu2 encodes AUX/IAA19, an auxin-regulated protein that functions together with the transcriptional activator NPH4/ARF7 to regulate differential growth responses of hypocotyl and formation of lateral roots in Arabidopsis thaliana. Plant Cell 16: 379-393.

Teh O, Moore I 2007 An ARF-GEF acting at the Golgi and in selective endocytosis in polarized plant cells. Nature 448: 493-496.

Tian Q, Reed JW 1999 Control of auxin-regulated root development by the Arabidopsis thaliana SHY2/IAA3 gene. Development 126: 711-721.

Xu M, Zhu L, Shou H, Wu P 2005 A PIN1 family gene, OsPIN1, involved in auxin-dependent adventitious root emergence and tillering in rice. Plant Cell Physiol. 46: 1674-1681.

Yang X, Lee S, So JH, Dharmasiri S, Dharmasiri N, Ge L, Jensen C, Hangarter R, Hobbie L, Estelle M 2004 The IAA 1 protein is encoded by $A X R 5$ and is a substrate of $\mathrm{SCF}^{\mathrm{TIR} 1}$. Plant J. 40: 772-782.

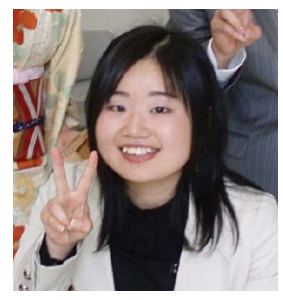

Yuka Kitomi studies on molecular mechanism of crown root formation in rice using some $\mathrm{crl}$ mutants. 


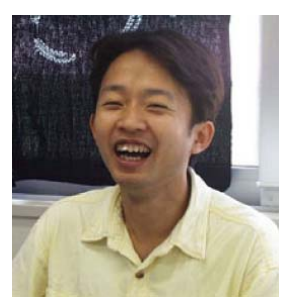

Dr. Atsushi Ogawa researches the root growth under the osmotic stress condition using the plant physiological and the histocytochemical methods.

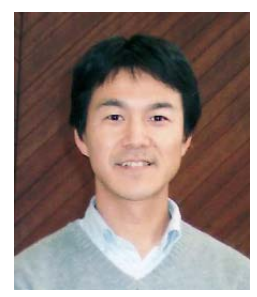

Dr. Yoshiaki Inukai tries to improve rice root system architecture through understanding of genetic mechanism regulating root formation in rice. 